\title{
LA EVALUACIÓN EN EL APRENDIZAJE COOPERATIVO: EL PESO DEL TRABAJO INDIVIDUAL DENTRO DEL TRABAJO EN EQUIPO
}

Evaluation in cooperative learning: the value of individual work in teamwork Avaliação de aprendizagem cooperativa: O valor do trabalho individual no trabalho em equipe

\section{Sandra Vázquez Toledo (1)}

\section{Cecilia Latorre Cosculluela (2)}

\section{Marta Liesa Orús (3)}

(1) Universidad de Zaragoza, España. Teléfono: +34 974239347. Correo electrónico: svaztol@unizar.es

(2) Universidad de Zaragoza, España. Correo electrónico: clatorre@unizar.es

(3) Universidad de Zaragoza, España. Correo electrónico: martali@unizar.es

\section{Resumen}

Si bien los procedimientos de aprendizaje cooperativo suponen estrategias cada vez más ampliamente utilizadas dentro de las aulas, surge la necesidad de ofrecer a los estudiantes de etapas educativas superiores correspondientes al ámbito universitario, la posibilidad de utilizar sistemas de calificación individual. Todo ello, con la finalidad de posibilitarles una diferenciación de sus aportaciones al grupo de aprendizaje cooperativo en cuanto a esfuerzo e implicación personal. En estetrabajo, se estudian y analizan los resultados obtenidos en un estudio planteado para tal fin, utilizando una metodología mixta que combina la utilización de instrumentos de recogida de datos cuantitativos (cuestionario) y cualitativos (grupos de discusión). Los resultados obtenidos evidencian considerables mejoras en la muestra de estudiantes universitarios participantes en el estudio en lo que respecta al apoyo mutuo manifestado en términos de aprendizaje entre iguales y refuerzo recíproco, además de una satisfacción general con este procedimiento de calificación individual dentro de los equipos de aprendizaje cooperativo, llevado a cabo mediante una coevaluación entre compañeros.

Palabras clave: aprendizaje cooperativo; calificación individual; implicación personal 


\begin{abstract}
Although cooperative learning procedures involve strategies that are widely used in classrooms, there is a need to offer students of higher educational stages the possibility of using individual qualification systems. All this, in order to enable them to differentiate their contributions to the cooperative learning group in terms of effort and personal involvement. In this work, we study and analyse the results obtained in a study designed for this purpose, using a mixed methodology that combines the use of quantitative and qualitative data collection instruments (questionnaire and discussion groups).The results evidenced considerable improvements in the sample of university students who participated in the study regarding mutual support expressed in terms of peer learning and reciprocal reinforcement, as well as general satisfaction with this individual qualification procedure within cooperative learning teams, carried out through peer evaluation.
\end{abstract}

Keywords: interactive

\title{
Resumo
}

Embora os procedimentos de aprendizagem cooperativa envolver cada vez mais amplamente estratégias utilizadas na sala de aula, a necessidade de proporcionar aos alunos com níveis educacionais mais elevados para a universidade, a possibilidade de utilizar qualificação individuais. Tudo isso, a fim de capacitá-los a diferenciarsuas contribuições para o grupo aprendizagem cooperativa, em termos de esforço e envolvimento pessoal. Neste estudo, eles são estudados e analisados os resultados de um estudo desenhado para o efeito, utilizando uma metodologia mista que combina a utilização de instrumentos de coleta de dados quantitativos (questionário) e qualitativas (focus groups). Os resultados mostram melhorias consideráveis na amostra de estudantes universitários com relação ao apoio mútuo expressos em termos de aprendizagem entre pares eo reforço mútuo, bem como a satisfação geral com este procedimento de classificação individual em equipes de aprendizagem cooperativa, conduzido por uma avaliação de pares entre os pares.

Palavras-chave: aprendizagem cooperativa; qualificação individual; envolvimento pessoal 


\section{Introducción}

Afortunadamente, la educación va desplazándose, cada vez más, hacia modelos colaborativos y cooperativos de enseñanza-aprendizaje centrados en el estudiante que buscan su implicación activa en las actividades y, es precisamente el aprendizaje cooperativo, la metodología que tiene mayor cabida en estos retos educativos (González \& Díaz, 2005). Este enfoque de enseñanza tiene claros efectos significativos sobre diversas áreas y, más específicamente, sobre la consideración y respeto a la diversidad entre los estudiantes (García, Traver, \& Candela, 2001). Los alumnos invierten sus esfuerzos en maximizar el aprendizaje de los componentes del equipo compartiendo sus recursos y asegurándose de que todos alcanzan las metas pre-establecidas.

En las técnicas de trabajo cooperativo que se basan en una estructura de trabajo interdependiente, las recompensas consisten en ofrecer premio al equipo que depende de las recompensas individuales. Así pues, los incentivos de logro se conceden en base al aprendizaje individual de cada uno de los miembros del equipo.

En esta línea, el presente estudio de investigación tiene por objetivo principal dar respuesta a la necesidad de calificación individual dentro de las formaciones de equipos de aprendizaje cooperativo en el ámbito universitario, así como analizar los resultados obtenidos con la aplicación de dicho procedimiento.

\section{Método}

El desarrollo del estudio se ha fundamentado en la utilización de un tipo de metodología mixta, en tanto que se ha pretendido alternar métodos cuantitativos y cualitativos. En un primer momento, se consideró y llevó a cabo el diseño de un cuestionario conformado por un total de 14 ítems para la recogida de datos, que fue administrado a una muestra de 125 estudiantes del Grado de Magisterio de Educación Infantil de la Universidad de Zaragoza comprendidos entre las edades de 18 y 24 años. El instrumento se ideó con la esencial finalidad de recoger información sobre las percepciones que estos estudiantes universitarios manifiestan acerca de la utilidad y eficacia de este sistema de calificación individual en el trabajo grupal.

En una segunda fase, se realizaron grupos de discusión que fueron utilizados para complementar la información de carácter cuantitativo recopilada en la primera fase de la investigación. Se trató, en todo momento, de asegurar un mínimo equilibrio en 
cuanto al género y número de participantes en ambos grupos, quedando así conformados dos grupos de discusión de 7 y 8 individuos. A grandes rasgos, la intención de estos grupos de discusión se relacionó con la necesidad de recogida de información de carácter cualitativo que complementase, de forma más trascendental, los datos recopilados mediante el cuestionario. Por tanto, la temática central de los grupos de discusión se focalizó en las opiniones más específicas que estos estudiantes declararon al respecto del beneficio de utilizar un sistema de calificación individual en los procesos de aprendizaje de carácter cooperativo.

\section{Resultados y discusión}

Tras la realización de los pertinentes análisis de la información obtenida por medio de los diferentes instrumentos de recogida de datos utilizados, se evidencian notables mejoras entre el aprendizaje de los estudiantes de este grado universitario participantes en el estudio. En cuanto a su proceso de aprendizaje, un 78\% del conjunto del alumnado indicaron el logro de una comprensión de determinados contenidos de la asignatura que, con una alta probabilidad, no habrían conseguido resolver de manera individual. Algunos de los estudiantes del grupo de discusión, incluso afirmaron lo siguiente: "este método nos ayuda a ir estudiando día a día. Muchos venimos de Bachiller, donde teníamos más seguimiento que en la Universidad, y con este método hemos ido estudiando día a día y también nos han ayudado a hacernos una idea de cómo pueden ser los exámenes finales”

Durante el tiempo que estos alumnos estudian juntos, comparten apuntes y resuelven dudas, se establecen entre ellos sistemas de intercambio de ayudas que conducen a una más elevada exigencia a nivel individual pues, según este procedimiento, el trabajo e inversión de esfuerzos de cada uno, contribuirá irremediablemente al éxito grupal (y viceversa).Ejemplo de ello, son las aportaciones que, algunos miembros de los grupos de discusión, indicaron afirmando lo siguiente: "Son muy útiles porque a la hora de realizar el examen ya te suena todo de haberlo estado trabajando previamente”.

Además de adquirir habilidades de autorregulación de su aprendizaje, los datos aportados por la muestra de participantes indican niveles más elevados en cuanto al compromiso individual y la responsabilidad personal. En consecuencia, estas capacidades lógicamente positivas provocan considerables mejoras de la motivación de 
los estudiantes ante el aprendizaje y actitudes más activas y comprometidas hacia las tareas encomendadas al equipo de trabajo. Reflejo de ello, es que un $75 \%$ de los estudiantes, reportaron un aumento de su interés por la asignatura. Todo ello, se deriva en manifiestas mejoras en cuanto a los resultados académicos de este alumnado.

\section{Conclusiones}

Queda demostrado, por tanto, que las estructuras de trabajo dentro de las aulas fundamentadas en el aprendizaje cooperativo, tienen una enorme influencia sobre las interacciones que se establecen entre los alumnos y sobre una serie de variables educativas como son el rendimiento académico y la autoestima, el proceso de socialización, la adquisición de competencias y destrezas sociales, y la promoción de valores sociales. Es imprescindible, no obstante, que cada Equipo Base asuma el compromiso de alcanzar sus objetivos, a la par que cada miembro debe comprometerse con las funciones y tareas que le haya correspondido.

\section{Referencias}

García, M.R., \& González, N. (2013). El aprendizaje cooperativo en la universidad. Valoración de los estudiantes respecto a su potencialidad para desarrollar competencias. Revista Iberoamericana para la Investigación y el Desarrollo Educativo, 4(7).

García, R., Traver, J. A., \& Cadela, I. (2001). Aprendizaje cooperativo. Fundamentos, características y técnicas. Madrid: CCS.ICCE.

González, G., \& Díaz, L. (2005). Aprendizaje colaborativo: una experiencia desde las aulas universitarias. Educación y educadores, 8, 21-44.

Johnson, D.W., \& Johnson, R.T. (1999). Making cooperative learning work. Theory into Practice, 38(2), 67-73. 